\title{
MECHANICAL DESIGN OF A PRECISION INSTRUMENT FOR MEASURING THE ROUNDNESS PROFILES OF MICRO SHAFTS
}

\author{
Neil Loychik, Mathieu Barraja, \\ Afzal Khan, R. Ryan Vallance* \\ Mechanical and Aerospace Engineering \\ George Washington University \\ 801 22nd St. NW, \\ Washington, DC, 20052 \\ Email: vallance@gwu.edu
}

\author{
Eric R. Marsh \\ Mechanical and Nuclear Engineering \\ Pennsylvania State University \\ 21 Reber Building, \\ College Station, \\ Pennsylvania, 16802 \\ Email: emarsh@psu.edu
}

\author{
Dave A. Arneson \\ Professional Instruments \\ 7800 Powell Road \\ Hompkins, \\ Minnesota, 55416 \\ Email: darneson@airbearings.com
}

\begin{abstract}
This paper presents a new instrument for measuring roundness profiles of axially symmetric micro shafts with diameters below 250 micrometers. The instrument will measure form error in cutting tools used for micro machining, small-hole metrology probes, and other high aspect ratio micro structures. This instrument operates on the same physical principle as scanning tunneling microscopes, which measure surface topography using fluctuations in tunneling of electrons between probe and sample. The instrument will measure roundness profiles using a fixedsensitive-direction arrangement of the detector, similar to macro scale metrology instruments. Challenges resulting from dimensional scaling necessitate an entirely new instrument compared to conventional instruments. We describe the need for this instrument, its working principle, architecture, the design of the traction drive mechanism, and the design of the nano positioning stages.
\end{abstract}

\section{INTRODUCTION}

Improvements in precision and accuracy was critical to achieving many technical products during the 19th and 20th centuries [1], and it will continue during the 21 st century with our emphasis on further miniaturization, microtechnology, and nanotechnology. Goods such as semiconductors, sensors, hard drives, micro electro mechanical systems (MEMS), and many

*Address all correspondence to this author. consumer products benefited from improved precision in manufacturing machinery, processes, and metrology. An advancement in manufacturing process or materials technology frequently requires a subsequent advance in metrology. Such is the case after the recent advances in 3D micro machining [2-4] and MEMS fabrication processes [5]. There is presently a need for more accurate and comprehensive dimensional metrology instruments and techniques. Furthermore, the metrology must be conducted at the nanometer scale so that reasonable precision (from 1 part per thousand towards 1 part per million) is achieved.

Figure 1 shows some components, fabricated by micro machining techniques, that will be suited for this new instrument. These components exhibit several common characteristics. First, they are made of electrically conductive materials such as tungsten, stainless steel, tungsten carbide, and polycrystalline diamond. The geometry of each component is principally symmetric about a centerline axis (axially symmetric). As a result, the dimensional metrology of these parts is principally associated with the roundness (or cylindricity) of the component. These components typically have diameters in the range of a few micrometers up to a few hundred micrometers, and their lengths may range up to millimeters. Therefore, these micro components exhibit high aspect ratios (length:diameter) up to 1:70.

Unfortunately, there is not presently an adequate method for accurately measuring the form (long spatial wavelengths), waviness (moderate spatial wavelengths), and roughness (short spatial wavelengths) over the entire surface of these parts. It is common 


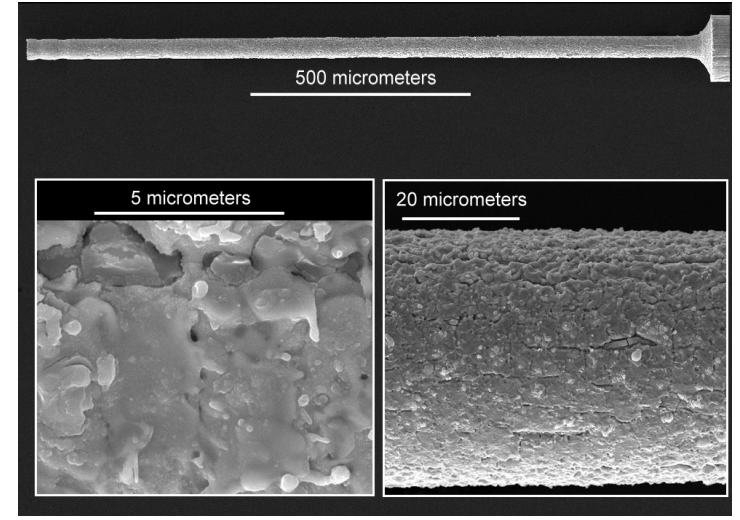

(a)

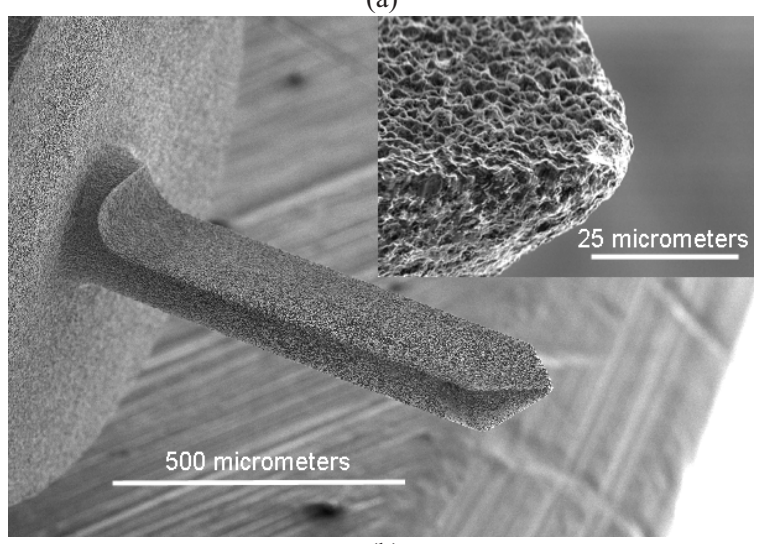

(b)

Figure 1. MICRO COMPONENTS EXHIBITING AXIAL SYMMETRY THAT REQUIRE NEW METROLOGY TECHNIGUE (a) HIGH ASPECT RATIO TUNGSTEN ELECTRODE USED IN MICRO ELECTRO DISCHARGE MACHINING (b) POLYCRYSTALLINE DIAMOND MICRO TOOL USED FOR MICRO MACHINING, FROM [6]

to use electron and optical microscopy for subjectively evaluating such components. More quantitatively, we also used scanning white light interferometry (SWLI) to scan segments of the surface and extract one-dimensional profiles along the length of shafts similar to that shown in Fig. 1(a), but it was not possible to acquire data around the entire cylindrical surface [7]. Therefore, there remains a need for determining geometric accuracy around the entire perimeter of the shaft.

Metrologists use two alternate approaches to assess out-ofroundness, which is the deviation from a perfect circular crosssection [8]. The first approach records a measured profile with a stationary gage (sensor or indicator) while rotating the sample on a precision spindle or rotary table. This is referred to as a fixed sensitive-direction measurement. In the second approach, known as a rotating sensitive-direction measurement, the profile of a stationary sample is recorded while the gage rotates. In both cases, the gage records fluctuations in the nominally circular shape of the sample's cross-section. Cylindricity is evaluated by repeating the measurements of the roundness profile with the gage positioned at several points along the sample's length.

Data from a roundness profile is a periodic function of the rotation angle of the sample (for fixed sensitive-direction measurements) or the gage (for rotating sensitive direction measurements). Therefore, the fluctuations in the radius $\Delta R$, which are the difference between the radius $R$ at orientation $\theta$ and the nominal radius $R_{o}$, can be represented as a Fourier series expansion as shown in Eq. (1), with each component having an amplitude $R_{k}$ and phase $\phi_{k}$.

$$
\Delta R(\theta)=R(\theta)-R_{o}=\sum_{k=2}^{n} R_{k} \cos \left(k \theta+\phi_{k}\right)
$$

This paper describes the mechanical design of a metrology instrument applicable to micro components such as those shown in Fig. 1. This new instrument will exceed the accuracy and precision requirements of similar instruments used for macro scale components. The instrument will measure the fluctuations in roundness profiles $\Delta R$ using fluctuations in tunneling current between the micro component and an electrochemically etched tungsten tip. The same method is used in scanning tunneling microscopy [9], which is capable of achieving atomic resolution on flat substrates [10].

\section{DESIGN SPECIFICATIONS OF THE INSTRUMENT}

The new instrument relies upon the working principle of a scanning tunneling microscope (STM). An STM scans an atomically-sharp metal tip, often made of tungsten or platinumiridium (Pt-Ir), across a rectangular region on the surface of a nominally flat substrate, as illustrated in Fig. 2. The dimensions of the rectangular region typically range from about $10 \times 10$ $\mathrm{nm}$ up to about $100 \times 100 \mu \mathrm{m}^{2}$. When the data is sampled for images (or contour plots) having 1024x1024 pixels or better, atomic-scale and nano-scale resolutions are achieved for small and large scan ranges, respectively.

An STM image results from topographically induced fluctuations in the current that flows between the sample and tip as the result of the quantum mechanical tunneling effect. A bias voltage $V$ between the tip and sample shifts the Fermi levels $E_{f}$ by an amount $\Delta E=e V$, where $e$ is the charge of an electron. The energy barrier between the two metals is approximately equal to the metals' work functions $\phi$. Since the electrons' wave functions decay exponentially across the barrier, it is probable that an electron will cross the barrier and current then becomes detectable. With a bias voltage $V$ of a few volts and a gap separation $s$ of $\sim 1 \mathrm{~nm}$, the tunneling current $i$ is typically on the order of $\sim 1$ 


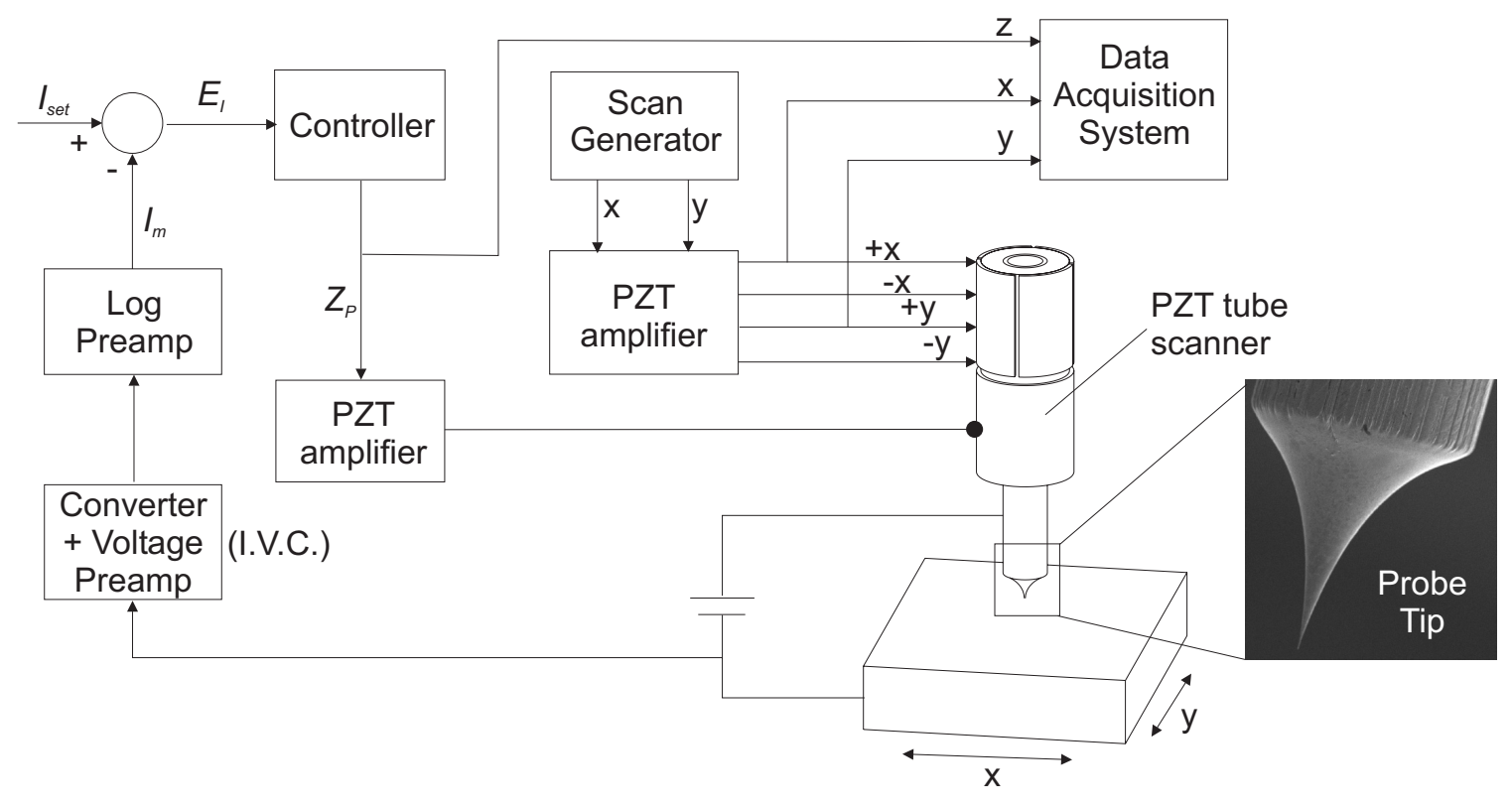

Figure 2. PRINCIPLE OF A SCANNING TUNNELING MICROSCOPE AND THE MEASUREMENT OF SURFACE TOPOGRAPHY, USING A SERVO CONTROL LOOP TO ADJUST THE POSITION OF THE PROBE TIP WHILE HOLDING THE TUNNELING CURRENT CONSTANT.

nA. For a fixed bias voltage $V$, the tunneling current depends exponentially on the gap separation $s$ as given in the approximate relation of Eq. 2. Due to the sensitivity of this exponential dependence, variations in the surface topography of around 0.001 nm are detectable. ${ }^{1}$

$$
i \approx V \exp \left\{-\left[\left(8 m_{e}\right)^{1 / 2} / h\right] \phi^{1 / 2} s\right\}
$$

Two distinct modes are used in scanning tunneling microscopy. The first mode does not adjust the position of the tip in the direction normal to the surface and simply records the tunneling current during the scanning motion tangent to the surface. Due to the exponential dependence of the current on the gap, this method is reserved for the smoothest surfaces, and so it is not appropriate for the micron-scale topography evident in most micro components [7]. The second mode uses a servo control loop to adjust the probe tip normal to the surface while holding the tunneling current constant during the scan motion. Then, the fluctuation of the surface topography is inferred by either measuring the displacement of the probe tip normal to the surface or via the product of the actuator voltage and the sensitivity of actuator. The second method is better suited to rougher surfaces, so

\footnotetext{
${ }^{1}$ Tunneling current is also sensitive to variations in the work function. For atomic-scale imaging, fluctuations in the tunneling current are actually the result of variation in the local density of states near the Fermi level.
}

it will be used in the new instrument.

The new instrument should fulfill performance specifications similar to those required for STMs, which have been described by Pohl [11]. The tip-to-sample position $s$ should be adjustable with picometer resolution, while the scanning directions should be controlled with a $10 \mathrm{pm}$ resolution. Course and fine positioning are required in the direction normal to the surface. Course positioning allows the probe tip to be moved into the range of the fine positioning stage. The course positioning in the tunneling direction should have a range of a few millimeters with about $0.1 \mu \mathrm{m}$ resolution. The fine positioning should have a range of a few micrometers with a resolution below $1 \mathrm{~nm}$. In addition, bandwidth of fine positioning should be on the order of $1 \mathrm{kHz}$.

Pohl [11] also recommends that the natural frequencies of the instrument structure exceed $100 \mathrm{~Hz}$ in order to minimize the sensitivity of the instrument to external vibrations. Then passive and active damping should be used to reduce amplitudes at the resonant frequencies. Thermal drift should be minimized by selecting materials with small coefficients of thermal expansion and having an appropriate architecture like a small structural loop and symmetry of the instrument. Finally, nonlinearities in actuators (e.g. hysteresis and creep in piezoelectric actuators) must be taken into account by operating at low electrical field, selecting elements with the highest possible sensitivity, and calibrating scanning motions.

The instrument must also provide motion to the sample. The most essential motion is the rotation of the sample by one com- 
plete revolution about its own axis. In addition, two linear motions are necessary to position the sample's centerline on the spindle's axis of rotation within a several nanometers. This degree of alignment is not necessary for macro-scale measurements of roundness profiles, but it becomes essential for nano scale accuracy of micro components. In the future, we will also incorporate a tip-tilt stage for adjusting $\theta_{x}$ and $\theta_{y}$ to align the centerline of the sample with the axis of rotation of the motorized spindle. This is essential for cylindricity measurements but less necessary for roundness. The adjustment range for this orientation is $\sim 1^{\circ}$ with fine resolution.

In summary, a total of five degrees of freedom are required for measuring roundness profiles on a micro shaft, and these are illustrated and identified in Fig. 3. In addition, the specifications for these motions are summarized in Table 1.

Table 1. MOTION SPECIFICATIONS FOR INSTRUMENT.

\begin{tabular}{l|c|c}
\multicolumn{1}{c|}{ Motion } & Range & Resolution \\
\hline Tip-to-sample position $x_{p}$ & $\sim 1 \mathrm{~mm}$ & $\sim 10 \mathrm{pm}$ \\
Scanning position $y_{p}$ & $\sim 5 \mu \mathrm{m}$ & $\sim 0.2 \mathrm{~nm}$ \\
Sample revolution $\theta_{z}$ & $360^{\circ}$ & $\sim 0.1^{\circ}$ \\
Tip and Tilt $\theta_{x}$ and $\theta_{y}$ & $\sim 1^{\circ}$ & $\sim 0.01^{\circ}$ \\
Sample positions $x_{p}$ and $y_{p}$ & $\sim 5 \mu \mathrm{m}$ & $\sim 2 \mathrm{~nm}$
\end{tabular}

\section{MECHANICAL DESIGN OF INSTRUMENT}

The architecture of the new instrument is illustrated in Fig. 4. The instrument consists of two principle sub assemblies, one of the sub assemblies manipulates the micro component (sample) and the other manipulates the probe tip. The micro component (sample) rotates slightly more than one revolution on a spindle that is indexed with a stepper motor through a traction drive. The stepper motor and traction drive are mounted below the spindle. A nano positioning stage is attached to the top of the air bearing spindle to provide motions $x_{s}$ and $y_{s}$ used to center the micro component on the axis-of-rotation. The micro component is held in a collet-style holder. A support structure symmetrically surrounds the spindle and sample and holds a second nano positioning stage that provides the probe motion in the $x_{p}$ and $y_{p}$ directions. A course positioning stage for the $z_{p}$ direction will be mounted on the probe's nanopositioning stage.

\section{Traction Drive and Compliant Motor Mount}

The instrument requires an extremely precise motor-driven spindle to establish an axis-of-rotation. Traditional rolling ele-

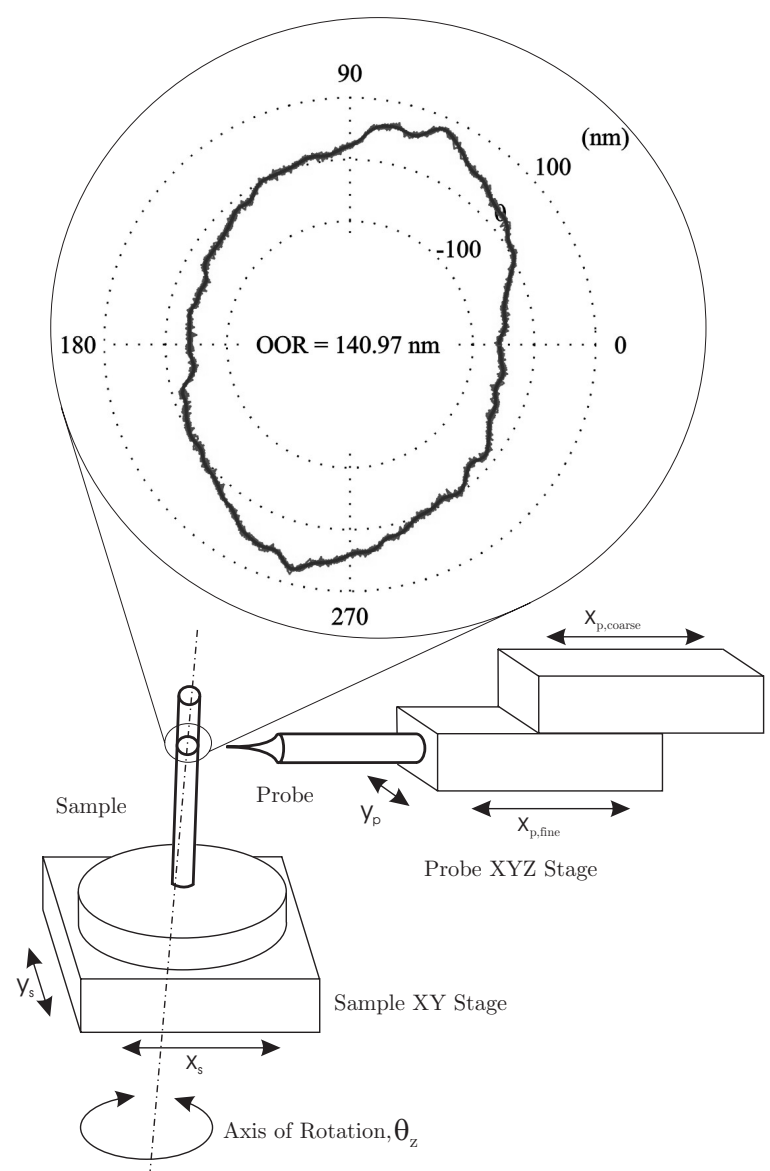

Figure 3. INSTRUMENT MOTIONS FOR FIXED-SENSITIVE DIRECTION MEASUREMENT OF ROUNDNESS PROFILES ON A MICRO SHAFT.

ment (ball or roller) bearings are not precise enough for this application. Instead, the instrument uses an externally pressurized aerostatic spindle (Professional Instruments, Model 4R Blockhead Spindle), which exhibit radial error motions of less than $10 \mathrm{~nm}$ [12]. These spindles consist of a rotor that is made of a hollow steel tube with two flange plates and a stator that encloses the perimeter of the rotor's central tube. Pressurized air (550-1034 $\mathrm{kPa})$ is supplied through the stator to a very small gap between the surfaces of the rotor and stator. Air flows out of the spindle between the annular gaps between the flange plates and stator. This produces high stiffness in the thrust, radial, and tilt directions.

Any motor driving an air bearing spindle introduces additional error motion due to servo loop chatter, magnetic pole print-through, imbalance, and other spindle-motor interactions. This is why the best spindle metrology is conducted without a motor while the spindle's rotor simply coasts at nearly constant speed $[12,13]$. In the new instrument, we attempt to minimize 


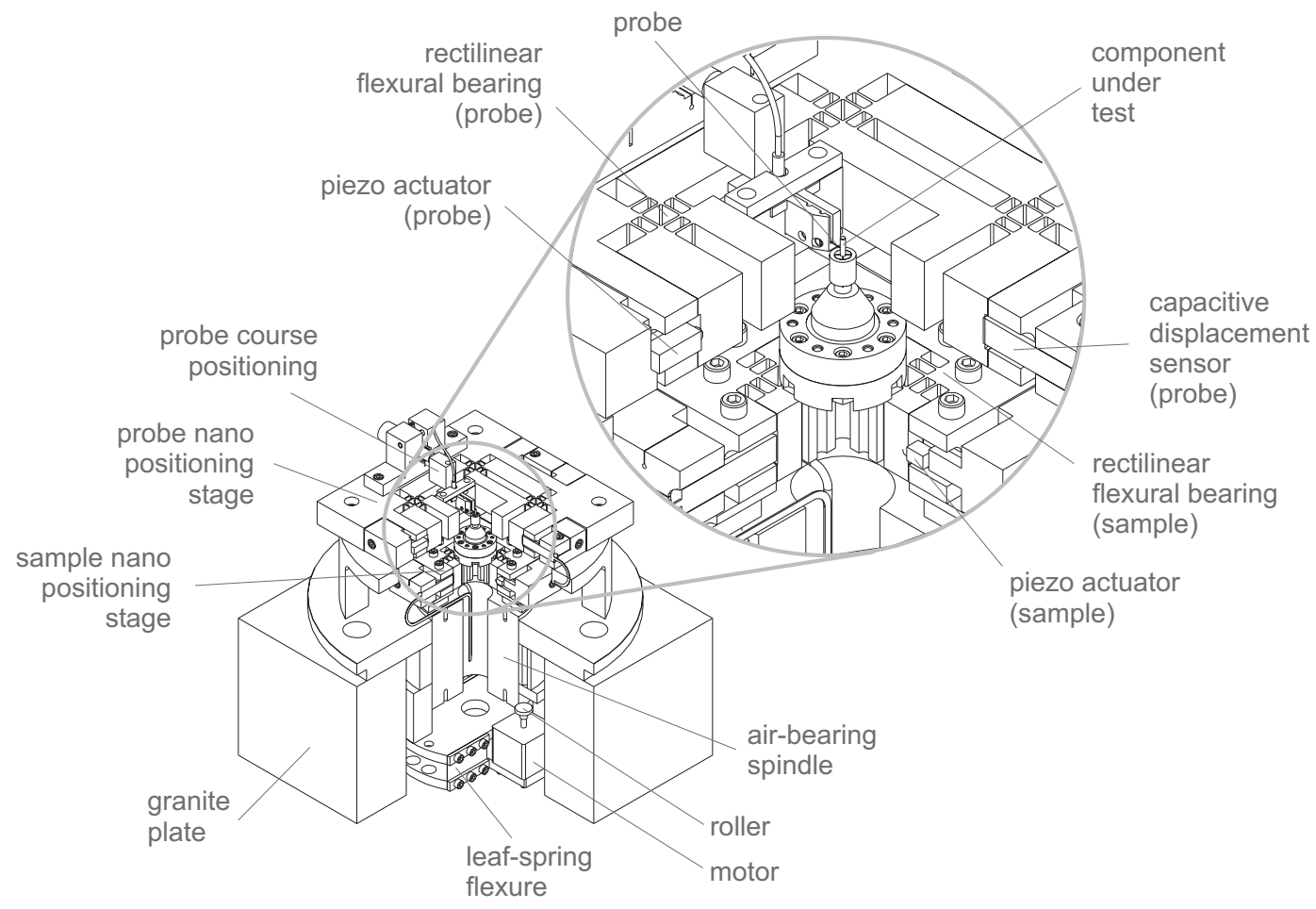

Figure 4. ARCHITECTURE OF THE INSTRUMENT, SHOWN WITH ONE QUARTER OF THE SOLID MODEL REMOVED TO REVEAL THE DETAILS THROUGH TO THE CENTERLINE (AXIS-OF-ROTATION) OF THE INSTRUMENT.

additional error motion from the drive system using the approach illustrated in Fig. 5. A rotary encoder, mounted to the spindle's bottom thrust plate, will enable spindle metrology with Donaldson reversal [14] and Estler reversal [15].

The spindle is driven through a traction drive (crowned roller pressed against the perimeter of the spindle rotor) by a stepper motor mounted on a compliant flexure. The traction drive provides a rolling point of contact, so a preload force is necessary to ensure that the roller remains in contact. A transmission ratio of about 10:1 is provided by the ratio of diameters for the spindle's thrust plate and the crowned roller.

The compliant flexure, formed by four parallel sheet metal springs, acts as a soft spring between the stepper motor and mechanical ground. The air gap supporting the spindle rotor is much stiffer than the compliant flexure. Therefore, radial error motion introduced from the stepper motor's shaft, bearings, or the roller causes the compliant flexure to move rather than the spindle rotor, minimizing the effect on the air bearing spindle's rotor.

The compliance of the flexure is adjustable using sheet metal with different thickness $w$ or modulus of elasticity $E$, while leaving their length $l$ and thickness $t$ fixed. The preload force is adjusted by advancing a screw that pushes against the moving platform of the flexure. This permits the roller to be completely disengaged from the spindle's thrust plate for coasting.

\section{Nano Positioning Stages}

A rectilinear nano positioning stage with two degrees of freedom $\left(x_{s}\right.$ and $\left.y_{s}\right)$ will be mounted on the top thrust plate of the air bearing spindle. The primary function of this nano positioning stage is to center the micro component's centerline on the spindle's axis-of-rotation within $\sim 50$ nanometers. Therefore this nano positioning stage requires a resolution of $\sim 2 \mathrm{~nm}$. This will be accomplished using the stage shown in the exploded view of Fig. 6.

The nano positioning stage will use a flexural (elastic) bearing $[16,17]$ that guides the rectilinear motion by elastic deformation (rather than rolling or sliding contact bearings) and essentially eliminates friction. We will use a bearing arrangement similar to one originally described by Holman et al. [18] that we improved for this application. The operation of the bearing is illustrated in Fig. 7(a). Thin beams bend to guide the central mass of the bearing so that it may only move in two orthogonal directions. The bearing is designed to have a static stiffness of $\sim 85$ $\mathrm{N} / \mu \mathrm{m}$ in both the $x_{s}$ and $y_{s}$ directions. As shown in Fig. 7(a), the resonant frequencies predicted by finite element analysis (FEA) all exceed $1 \mathrm{kHz}$, which is recommended for scanning tunneling microscopy [11]. The central mass of the bearing displaces when two piezoelectric stack actuators (Piezomechanik) press against the intermediate bodies. The motion of the central body is de- 

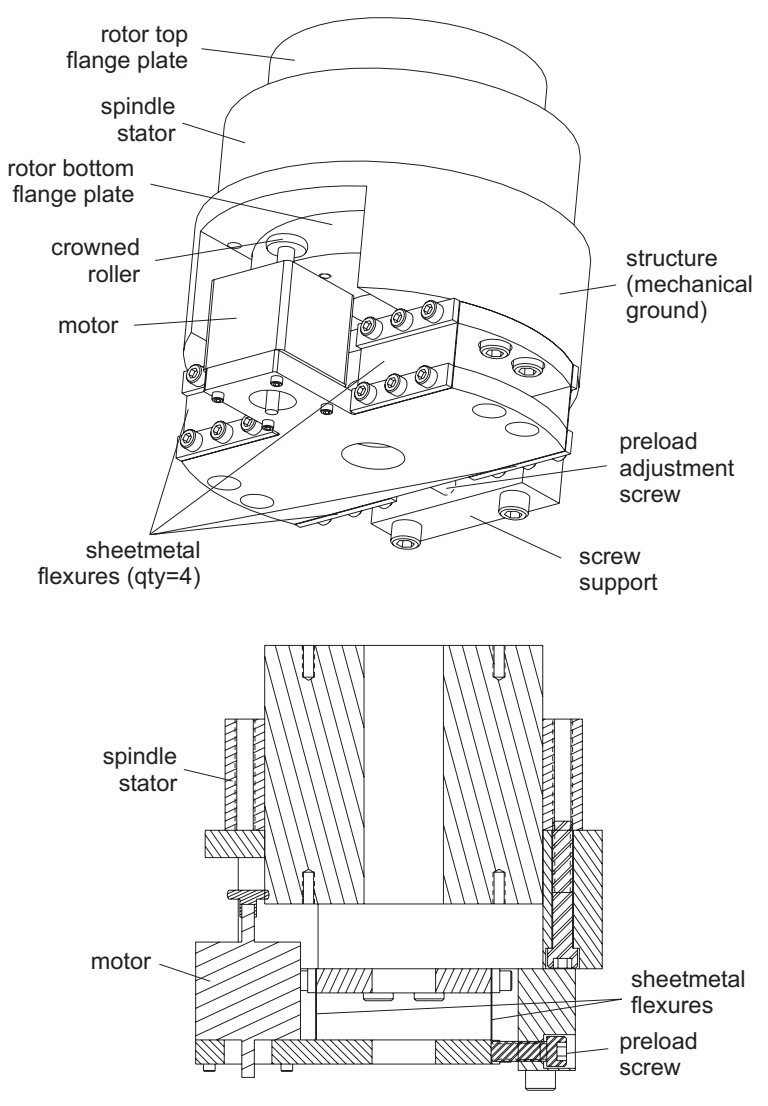

Figure 5. TRACTION DRIVE SYSTEM

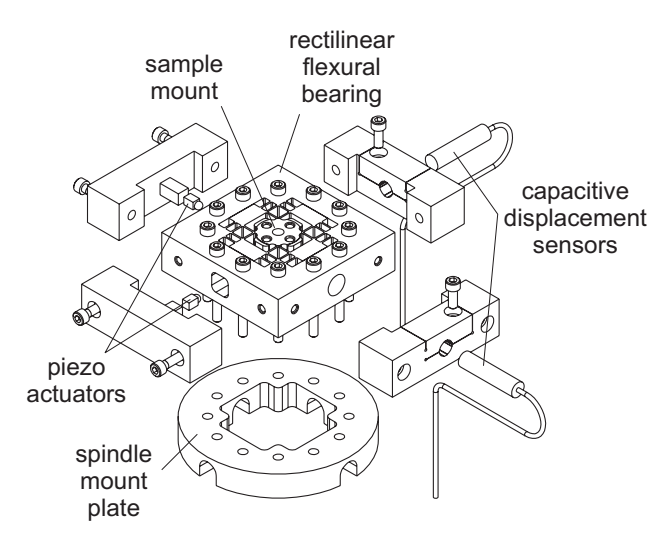

Figure 6. NANO POSITIONING STAGE FOR CENTERING MICRO SHAFT ON AXIS OF ROTATION.

tected by two non-contact capacitive displacement sensors (Lion Precision) that target the intermediate bodies opposite each actuator. These sensors are limited to resolutions of $\sim 2 \mathrm{~nm}$, which is adequate for centering the sample on the axis of rotation.

A second nano positioning stage with two degrees of free-
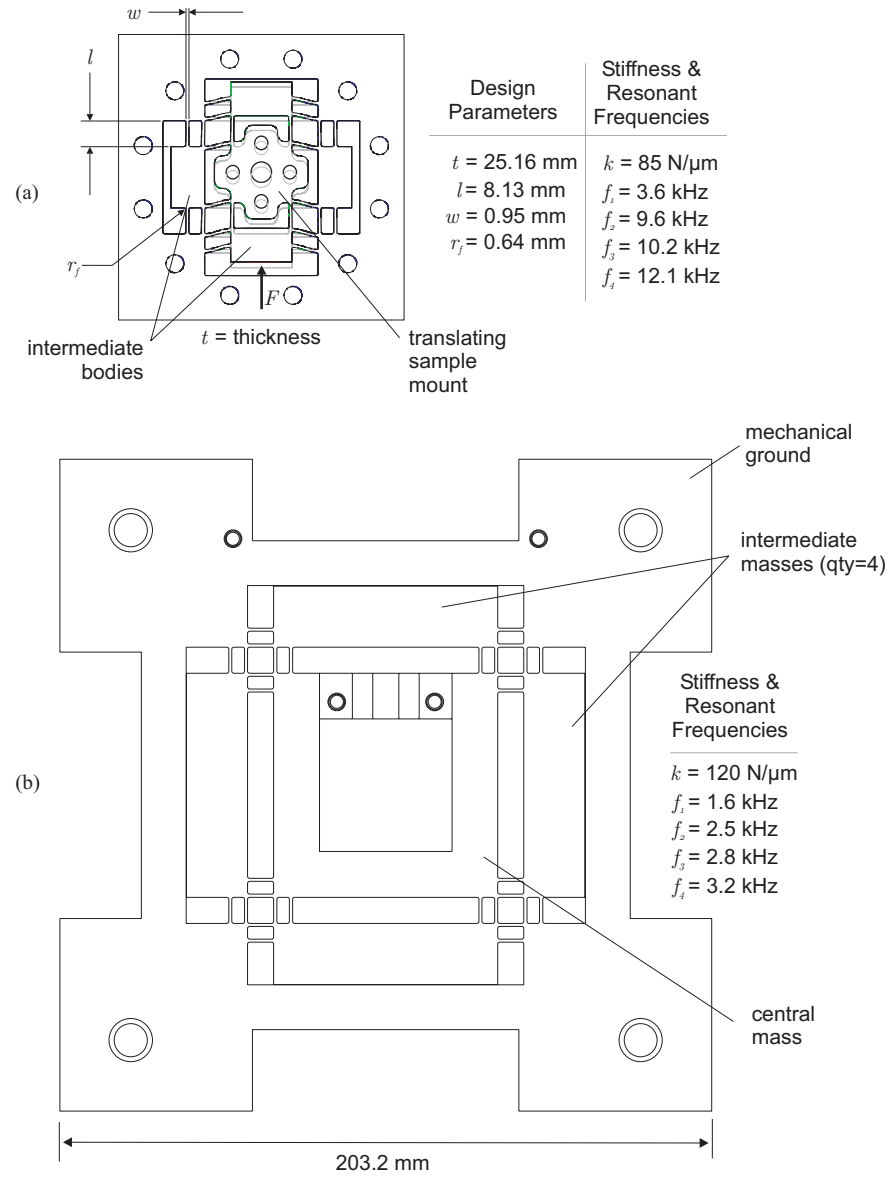

Figure 7. RECTILINEAR, TWO DEGREE-OF-FREEDOM, FLEXURES FOR NANO POSITIONING STAGES (A) FLEXURE FOR CENTERING SAMPLE ON AXIS-OF-ROTATION AND (B) FLEXURE FOR POSITIONING PROBE TIP

dom, similar to the one for the sample, will be used for moving the probe tip in the $x_{p}$ and $y_{p}$ directions. This nano positioning stage, illustrated in Fig. 8, will consist of a rectilinear flexural bearing, another set of piezoelectric actuators, and set of capacitive displacement sensors. At least $10 \mu \mathrm{m}$ and $5 \mu \mathrm{m}$ of travel will be provided in the $y_{p}$ direction and $x_{p}$ directions, respectively.

With assistance from Professional Instruments Co., we fabricated a prototype of this nano positioning stage. In a recent test, we excited the piezoelectric actuators with an open-loop +/$10 \mathrm{mV}$ signal and observed just less than a $2 \mathrm{~nm}$ amplitude of displacement. Furthermore, the results clearly demonstrated a resolution (minimum step size) far below $1 \mathrm{~nm}$. We also tested the travel range of the stage with $150 \mathrm{~V}$ square wave and found that the travel range is $\sim 5.5 \mu \mathrm{m}$, which is adequate for expected outof-roundness values of $\sim 1 \mu \mathrm{m}$. 


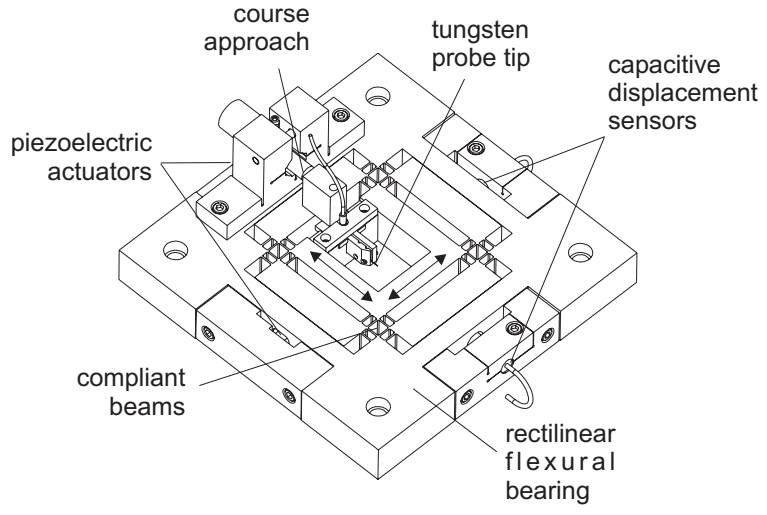

Figure 8. Nanopositioning stage for moving the metallic probe tip in the $x_{p}$ and $y_{p}$ directions. The stage will be guided by a rectilinear flexural bearing and actuated by piezoelectric actuators. A laser interferometer will be used to measure the displacement of the probe tip in the $x_{p}$ direction, while a capacitive displacement sensor will be used in the $y_{p}$ direction.

\section{CONCLUSION}

This paper describes a new instrument suitable for measuring the form, waviness, and roughness of axially symmetric micro components. We are presently limited to either visual inspection by optical or electron microscopy (as used in Fig. 1), surface profiles from interferometry, or scanning probe microscopy on nominally flat surfaces. The new instrument will enable the resolution and accuracy associated with the scanning probe techniques to be achieved with $3 \mathrm{D}$ micro components. Important aspects in the new instrument include a precision axis of rotation with a traction drive system and two nano positioning stages. One nano positioning stage positions the sample on the axis of rotation, so it is mounted on the rotating thrust plate of the spindle. The second aligns the probe tip to the sample and adjusts the separation between the probe tip and the sample. The design of the instrument is complete, and it is currently being assembled and tested. Initial measurements from the instrument are scheduled for late 2006.

\section{ACKNOWLEDGMENT}

We acknowledge Professional Instruments Co. for contributions during design and also fabricating this the instrument.

\section{REFERENCES}

[1] Evans, C., 1989. Precision Engineering: An Evolutionary View. Cranfield Press.

[2] Masuzawa, T., and Tönshoff, H. K., 1997. "Threedimensional micromachining by machine tools". Annals of the CIRP (International Institution for Production Engineering Research), 46, pp. 621-628.
[3] Masuzawa, T., Fujino, M., Kobayashi, K., and Suzuki, T., 1985. "Wire electrodischarge grinding for micromachining". Annals of the CIRP (International Institution for Production Engineering Research), 34, pp. 431-434.

[4] Masuzawa, T., and Fujino, M., 1990. A process for manufacturing very fine pin tools. SME Technical Paper MS90307, Society of Manufacturing Engineers, Int. Manufacturing Technology Conf., Chicago, IL, September.

[5] Madou, M. J., 1998. Fundamentals of Microfabrication. CRC-Press.

[6] Morgan, C. J., Vallance, R. R., and Marsh, E. R., 2004. "Micro machining glass with polycrystalline diamond tools shaped by micro electro discharge machining". Journal of Micromechanics and Microengineering, 14(12), December, pp. 1687-1692.

[7] Vallance, R. R., Morgan, C. J., and Marsh, E. R., 2004. "Micro-tool characterization using scanning white light interferometry". Journal of Micromechanics and Microengineering, 14(8), August, pp. 1234-1243.

[8] ASME, 1972. ASME/ANSI B89.3.1 Measurement of outof-roundness. American Society of Mechanical Engineers (ASME).

[9] Binnig, G., Rohrer, H., Gerber, C., and Weibe, E., 1982. "Surface studies by scanning tunneling microscopy". Physical Review Letters, 49(1), July, pp. 57-61.

[10] Binnig, G., and Rohrer, H., 2000. "Scanning tunneling microscopy". IBM J. Res. Develop., 44(1/2), January/March, pp. 279-293.

[11] Pohl, D. W., 1986. "Some design criteria in scanning tunneling microscopy". IBM J. Res. Develop., 30(4), July, pp. 417-427.

[12] Grejda, R., Marsh, E. R., and Vallance, R. R., 2005. “Techniques for calibrating spindles with nanometer error motion". Precision Engineering, 29(1), January, pp. 113-123.

[13] Marsh, E. R., Couey, J., and Vallance, R. R., 2005. "Comparison of three spindle error motion separation techniques". In Proceedings of the Annual Meeting of the American Society for Precision Engineering.

[14] Donaldson, R., 1972. "A simple method for separating spindle error from test ball roundness error". CIRP Annals, 21(1), pp. 125-126.

[15] Salisbury, J. G., 2003. "Implementation of the estler face motion reversal technique". Precision Engineering, 27, pp. 189-194.

[16] Paros, W., and Weisbord, L., 1966. "How to design flexure hinges". Machine Design, 27, pp. 151-156.

[17] Smith, S. T., 2000. Flexures: Elements of Elastic Mechanisms. CRC Press.

[18] Holman, Laman, Scholte, Heerens, and Tuinstra, 1996. "A calibrated scanning tunneling microscope equipped with capacitive sensors". Review of Scientific Instrments, 67(6), June, pp. 2274-2280. 